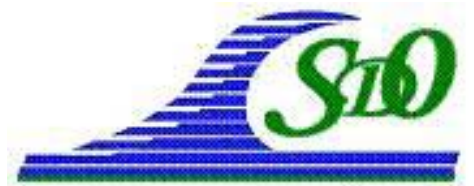

XI ${ }^{\text {èmes }}$ Journées Nationales Génie Côtier - Génie Civil

Les Sables d'Olonne, 22-25 juin 2010

DOI:10.5150/jngcgc.2010.051-V @ Editions Paralia CFL

disponible en ligne - http://www.paralia.fr - available online

\title{
Monitoring d'un rechargement massif : la plage de Carnon (Languedoc-Roussillon)
}

\author{
Cyril VANROYE ${ }^{1}$, Benoît BARTHAZ ${ }^{1}$
}

1. Direction Régionale de l'Environnement, de l'Aménagement et du Logement du Languedoc-Roussillon

Service des Espaces Littoraux, Unité Aménagements et Risques Littoraux

520 allée Henri II de Montmorency, 34064 Montpellier Cedex 2, France

cyril.vanroye@developpement-durable.gouv.fr ;

benoit.barthaz@developpement-durable.gouv.fr

\section{Résumé :}

Dans le cadre de l'opération d'aménagement et de protection du littoral du Golfe d'Aigues-Mortes, un rechargement massif a été mis en oeuvre. Plus de 1 million de $\mathrm{m}^{3}$ de sables ont été mobilisés pour engraisser quatre plages. Les travaux ont été réalisés de janvier à avril 2008.

Sur la commune de Carnon, deux secteurs ont été rechargés : la plage de Carnon Est qui est équipée d'ouvrages de protection (épis) et la plage du lido du Petit Travers et du Grand Travers qui est une plage naturelle. Au total, ces plages ont été rechargées par $565000 \mathrm{~m}^{3}$ de sable sur une distance de $5100 \mathrm{~m}$. Ce rechargement a été réalisé par une Drague Aspiratrice en Marche (D.A.M.) et localisé en haut de plage.

Le monitoring mis en place depuis la réalisation des travaux par la Direction Régionale de l'Environnement, de l'Aménagement et du Logement du Languedoc Roussilon consiste en un suivi régulier du trait de côte par DGPS, des levés topobathymétriques annuels entre le haut de plage et l'isobathe $-10 \mathrm{~m}$ ainsi que des prélèvements et analyses granulométriques suivant des profils perpendiculaires au rivage.

Les résultats acquis lors de la première année suivant le rechargement mettent en évidence :

- le recul court terme important de la plage rechargée,

- la migration du sédiment perpendiculairement dans le profil et l'engraissement préférentiel de la barre externe.

Le bilan quantitatif reste bénéficiaire pour les secteurs rechargés.

Mots-clés :

Rechargement - Monitoring - Dynamique sédimentaire

\section{Introduction}

Le littoral du Languedoc-Roussillon est de nature essentiellement sableuse sur près de $200 \mathrm{~km}$. Il a fait l'objet dans les années 60 d'une opération d'aménagement d'envergure dans le cadre de la Mission d'Aménagement Touristique du Littoral Languedoc- 
Roussillon dite Mission Racine. L'accroissement des enjeux sur la frange littorale associé à une dynamique sédimentaire intense a entraîné la mise en place d'ouvrages de protection dits lourds du type brise-lames et épis. Au nombre de 200 sur la façade languedocienne, ces ouvrages n'ont pour autant pas enrayé le phénomène (SAMAT, 2007). La recherche de solutions basées sur le fonctionnement naturel du littoral (MIALR et al., 2003) a incité à retenir dans le cadre de l'opération d'aménagement et de protection des communes littorales du Golfe d'Aigues-Mortes la technique du rechargement massif (beachnourishment). L'objet du travail qui suit est de présenter les éléments issus du suivi morphologique mis en place par la Direction Régionale de l'Environnement, de l'Aménagement et du Logement du Languedoc-Roussillon.

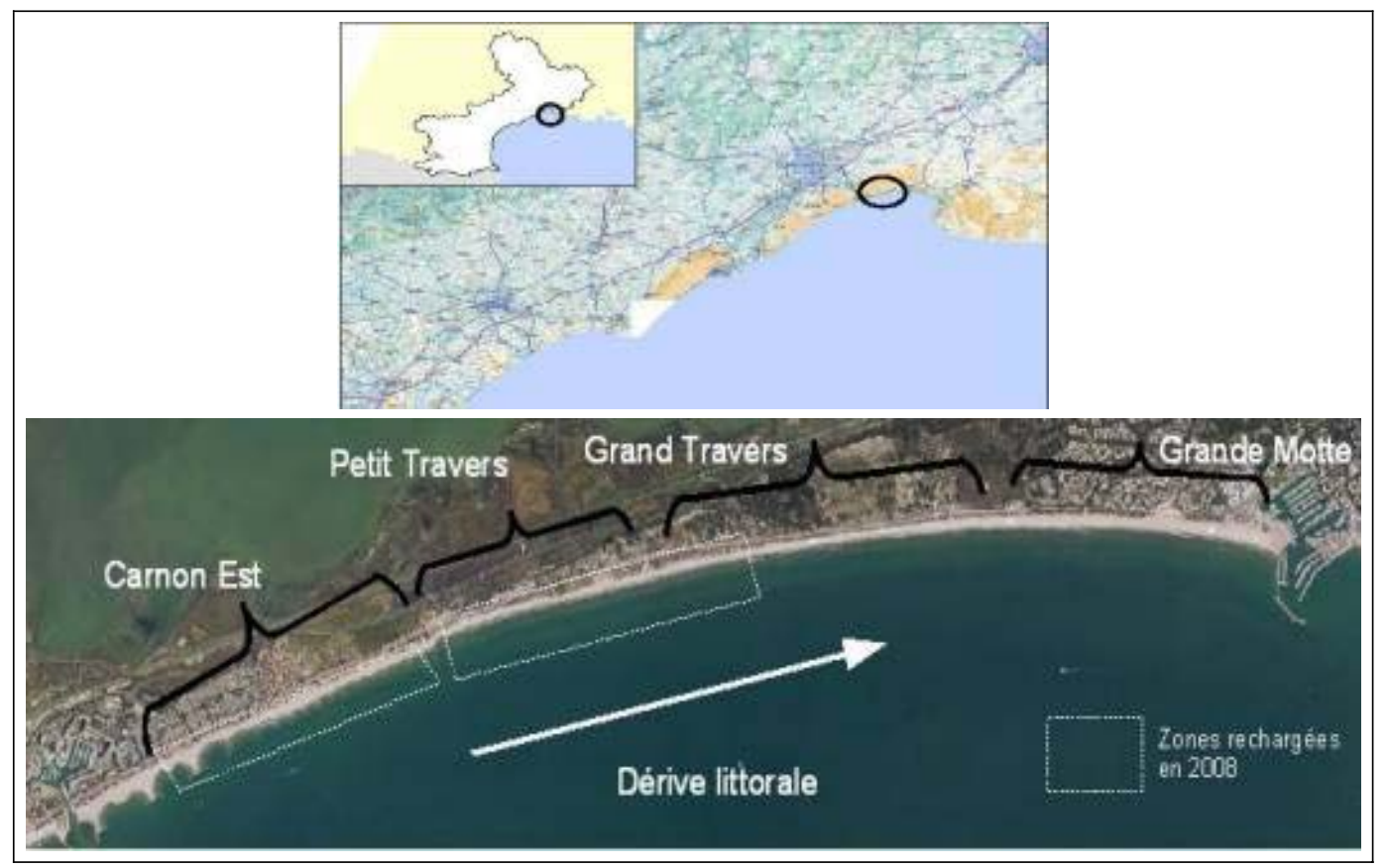

Figure 1. Localisation et présentation générale du site d'étude.

\section{Le site d'étude}

Le site d'étude est le littoral de la commune de Carnon dans le département de l'Hérault. Ce littoral, situé dans la partie centrale du Golfe d'Aigues-Mortes, constitue une souscellule sédimentaire (port de Carnon-port de la Grande Motte) et est intégré à la cellule sédimentaire délimitée à l'Ouest par une digue fluviale sur la commune de Frontignan et les installations portuaires de Port-Camargue. Il est affecté d'une dérive littorale orientée vers l'Est qui transporterait entre 25000 et $30000 \mathrm{~m}^{3}$ de sédiments (SOGREAH, 2003). Les sites considérés s'étendent sur une distance de $5100 \mathrm{~m}$ et sont : Carnon-Est, zone équipée d'ouvrages (brise-lames et épis) et la plage du Petit et du Grand Travers, zone naturelle. Le secteur est en érosion avec un recul moyen du trait de côte de 0,6 m à 0,8 m par an sur la période 1992-2002 (SOGREAH, 2003) à Carnon et de 1,4 m par an 
au Travers. Le rechargement massif réalisé de janvier à avril 2008 a consisté à injecter en haut de plage $190000 \mathrm{~m}^{3}$ à Carnon-Est et $375000 \mathrm{~m}^{3}$ sur la plage du Travers.

Les sédiments superficiels des plages sont constitués de sables fins présentant un diamètre médian compris entre $230 \mu \mathrm{m}$ et $260 \mu \mathrm{m}$. Les sables issus du site d'extraction et utilisés pour le rechargement présentent un diamètre médian compris entre $170 \mu \mathrm{m}$ et $190 \mu \mathrm{m}$. Cette différence de granulométrie a nécessité un volume d'apport plus important que les volumes estimés en besoins. L'utilisation de la méthodologie du Coastal Engineering Manual a conduit a des facteurs de sur-rechargement compris entre 1,15 et 1,50 .

\section{Méthodologie}

Le suivi mis en place est constitué de relévé du trait de côte réalisé au DGPS par la DREAL LR sur la plage du Travers et de relevés topo-bathymétriques réalisés par l'EID Méditerranée dans le cadre d'un contrat avec la DREAL LR. La situation après travaux résulte des résultats des levés topo-bathymétriques réalisés dans le cadre du chantier de rechargement immédiatement après les travaux et ayant servi au constat de la situation finale sur les secteurs rechargés. Les profils de suivi après rechargement couvrent les secteurs rechargés mais aussi le secteur à l'aval du transit jusqu'à la Grande-Motte. Ils sont distants de $25 \mathrm{~m}$ pour la partie topographique et de $30 \mathrm{~m}$ en bathymétrie.

L'exploitation des traits de côte a été réalisée suivant des transects espacés de $100 \mathrm{~m}$ ( au nombre de 60 à l'échelle de la plage naturelle) et pour lesquels le recul de la ligne du rivage a été calculé pour chaque période disponible. Les dates de réalisation des levés après travaux sont : 17 mars 2008, 16 avril 2008, 10 juin 2008, 17 septembre 2008, 4 mars 2009, 18 mai 2009, 22 septembre 2009. Les relevés topo-bathymétriques ont été convertis en grilles afin de constituer des Modèles Numériques de Terrain (M.N.T.) au moyen du logiciel ENGAGE3D. Ces M.N.T. ont permis de calculer des variations de volume en comparant la situation immédiatement après rechargement (avril 2008) et la situation un an après travaux sur les zones rechargés (juillet 2009).

\section{Résultats}

\subsection{Analyse de l'évolution des traits de côte}

Afin de connaître la variation sur une année et de ne pas se situer trop près de la fin du rechargement, les variations entre septembre 2008 et septembre 2009 sont considérées. Cette comparaison met en évidence des variations importantes suivant trois compartiments d'Ouest en Est dans le sens de la dérive littorale (figure 1) :

- le secteur du Petit Travers où le recul est très fort (le recul moyen est de $-12 \mathrm{~m}$ ),

- le secteur du Grand travers où l'érosion est forte (le recul moyen est de $-6 \mathrm{~m}$ sur la même période), 
Thème 2 - Dynamique sédimentaire et transports des particules

- le secteur de la Grande Motte où la plage est quasiment stabilisée (le recul moyen est de $-2 \mathrm{~m})$.

Afin de préciser le comportement saisonnier, l'exploitation des traits de côte disponibles a été réalisée sur les secteurs mis en évidence précédemment en considérant des situations sortie d'hiver, sortie de printemps et sortie d'été (voir figure 2).

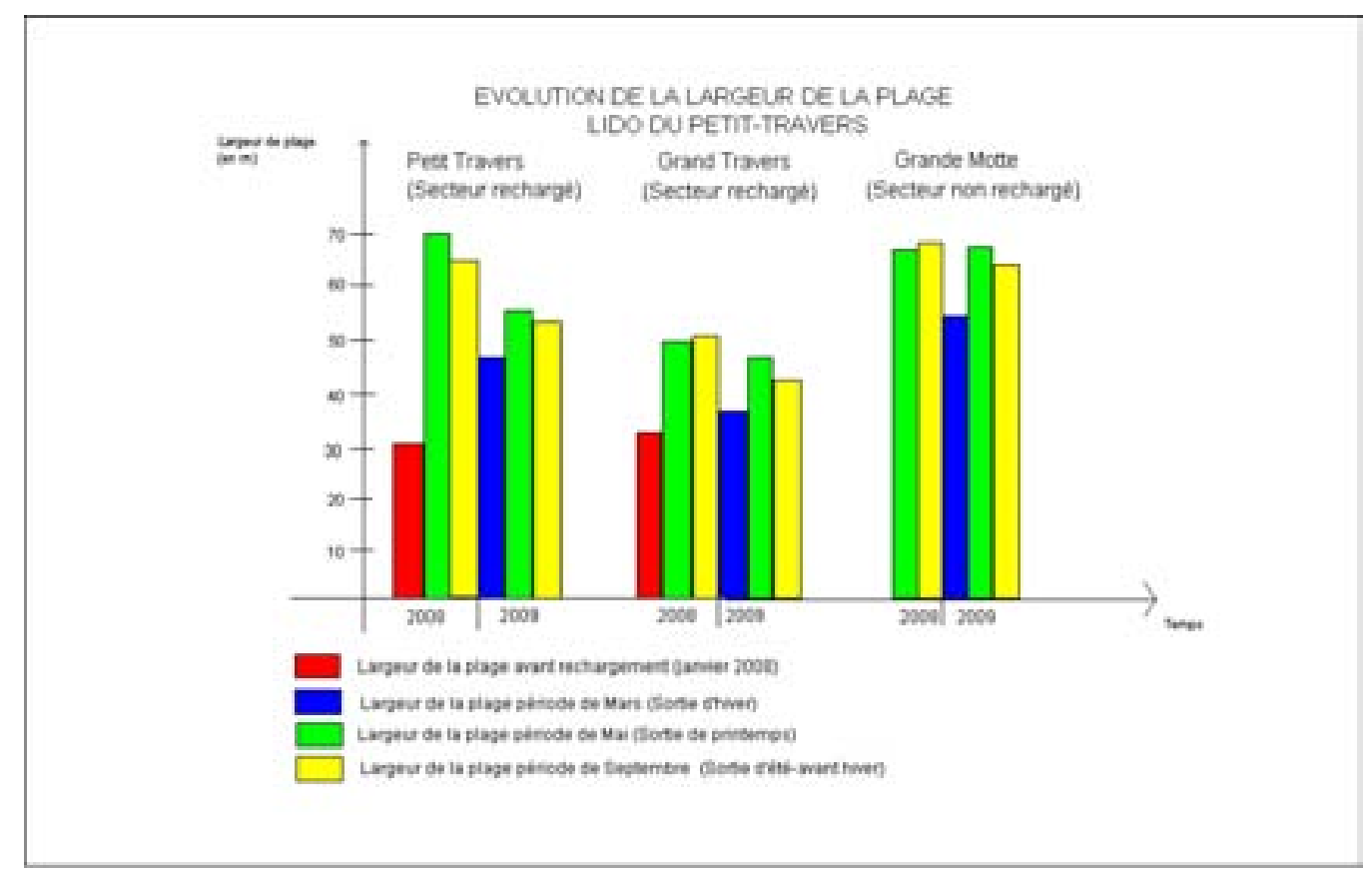

Figure 2. Evolution saisonnière de la largeur de plage à partir de l'analyse des traits de côte après rechargement.

\subsection{Les variations de volume}

Les mouvements dans le profil sont assez nets (voir figure 3). Le rechargement réalisé en haut de plage a d'ores et déjà migré dans le profil en engraissant la barre externe. On constate des variations quasi-nulle au-delà de l'isobathe $-4 \mathrm{~m}$ (profondeur de fermeture). Pour les secteurs considérés, les variations de volume sont :

Tableau 1. Variations de volume.

\begin{tabular}{lll}
\hline Secteur & Variation de volume 2008-2009 & Volume rechargé \\
\hline Carnon Est & $-43686 \mathrm{~m}^{3}$ & $190000 \mathrm{~m}^{3}$ \\
Petit Travers & $+46133 \mathrm{~m}^{3}$ & $375000 \mathrm{~m}^{3}$ \\
\hline
\end{tabular}




\section{XI ìmes Journées Nationales Génie Côtier-Génie Civil}

Les Sables d'Olonne, 22-25 juin 2010

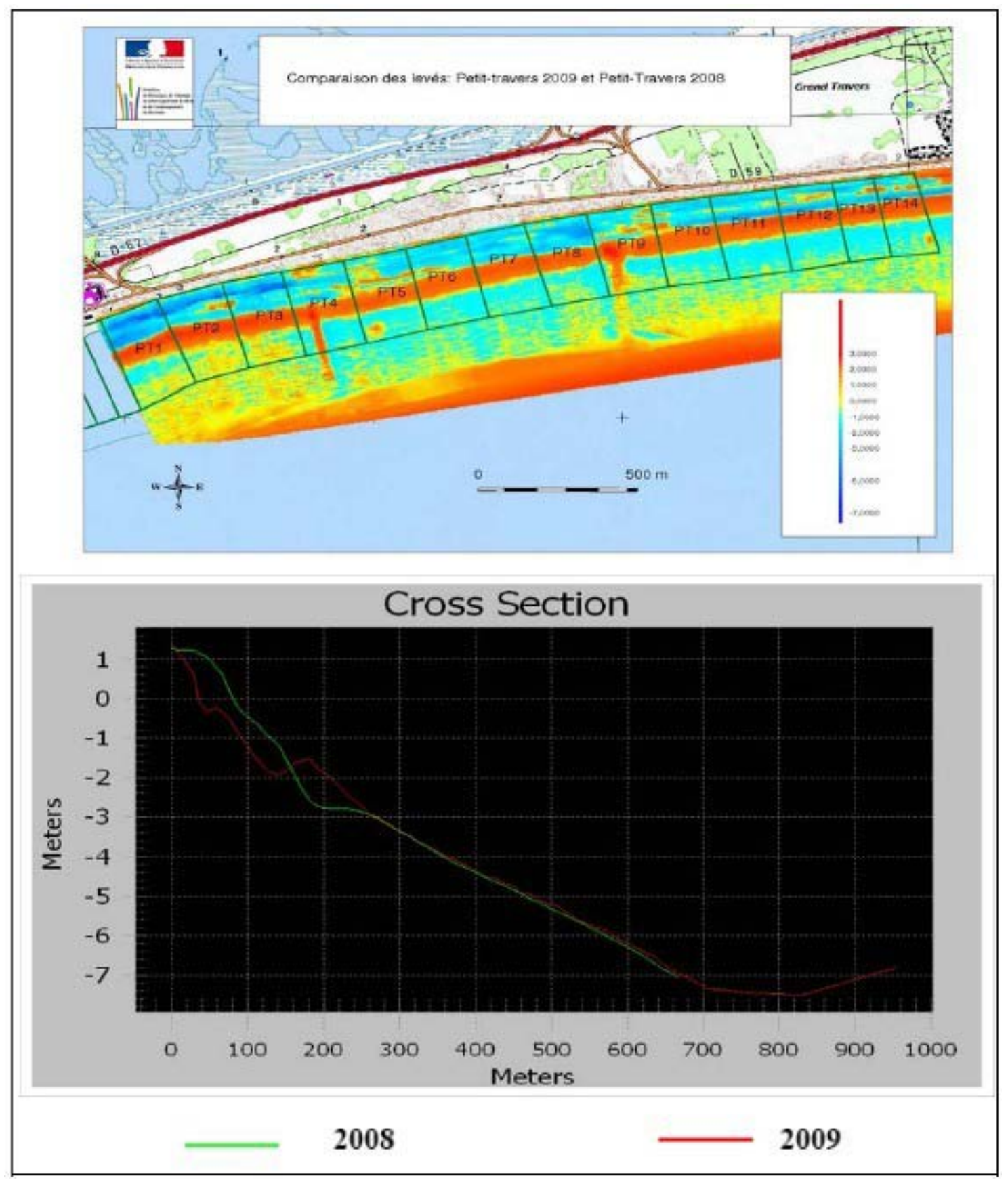

Figure 3. Evolution du rechargement sur le secteur du Petit Travers (en haut : à

l'échelle de la zone rechargée, en bas : suivant un profil transversal).

\section{Discussion et conclusion}

L'analyse des traits de côte montre des variations très marquées de recul la première année. Ces variations sont à mettre en relation avec la technique de rechargement qui a consisté à recharger la plage émergée. Ces reculs sont cependant plus importants que ceux estimés avant le rechargement. Le recul évalué entre 1992 et 2002 (SOGREAH, 
2003) était de l'ordre de 30 à $35 \mathrm{~m}$ sur le secteur du Petit Travers soit $3 \mathrm{~m}$ par an. Cela incite donc à émettre plusieurs hypothèses : l'état d'équilibre n'est pas encore atteint ou compte tenu de la granulométrie plus fine du sable mis en place par rapport au sable d'origine une augmentation de la vitesse d'érosion. L'analyse des variations saisonnières et notamment la comparaison des largeurs de plage sortie d'hiver et sortie d'été montre cependant un retour à une largeur de plage quasi équivalente en sortie d'été et en sortie d'hiver (figure 2).

Les variations dans le profil sont celles attendues avec un rechargement naturel de la barre externe (élévation de la crête de près de $60 \mathrm{~cm}$ ) dans l'année qui suit le rechargement. Les volumes perdus ou gagnés sur chaque secteur sont supérieurs aux estimations de la dérive littorale 25000 à $30000 \mathrm{~m}^{3}$ (SOGREAH, 2003) même si on tient compte du facteur de sur-rechargement. Ces valeurs incitent à considérer que l'état d'équilibre n'est pas encore atteint alors qu'une grande partie des déplacements de sable dans le profil ont eu lieu.

Les deux zones d'accumulation préférentielles (figure 3) perpendiculairement à la plage sont dues au positionnement des siphons utilisés pour réalisés le rechargement.

D'autres suivis post-rechargement (PARK et al., 2009) ont permis de mettre en évidence la complexité de l'évolution des rechargements avec notamment l'exportation vers le large de sédiments qui n'apparaît pas le cas héraultais.

Les analyses granulométriques réalisées font l'objet d'un traitement statistique basé sur la méthode GSTA (Gran Size Trend Analysis) pour affiner l'approche sur le transport sédimentaire.

\section{Références bibliographiques}

MIALLR, SMNLR, BRL, EID -Mission interministérielle d'aménagement du littoral du Languedoc-Roussillon, Service Maritime et de Navigation du Languedoc Roussillon, BRL ingénierie, Entente Interdépartementale pour la Démoustication- (2003). Orientations stratégiques pour la gestion de l'érosion en Languedoc-Roussillon, 24 p, [URL http://www.languedoc-roussillon.pref.gouv.fr/grandsdossiers/missionlittoral/etudes_rapport.shtm ] PARK J.-Y., GAYES P.T., WELLS J.T. (2009), Monitoring Beach Renourishment alaong the Sediment-Starved Shoreline of Grand Strand, South Carolina. Journal of Coastal Research, 25(2), pp 336-349. doi:10.2112/07-0929.1

SAMAT O. (2007). Efficacité et impact des ouvrages en enrochements sur les plages microtidales. Le cas du Languedoc et du delta du Rhône. Thèse de doctorat, Université de Provence Aix-Marseille 1.

SOGREAH (2003). Etudes générales pour la protection et l'aménagement du Golfe d'Aigues-Mortes. Rapport. 